\title{
Resíduo industrial como matéria-prima alternativa para a produção de filtros cerâmicos refratários
}

\section{(Industrial waste as alternative raw material for the production of refractory ceramic filters)}

\author{
A. C. Carvalho ${ }^{1}$, F. Raupp-Pereira ${ }^{2 *}$, J. B. Rodrigues Neto ${ }^{2}$, A. P. Novaes de Oliveira ${ }^{2}$ \\ ${ }^{1}$ Mestrado em Engenharia de Processos - MEP, Universidade da Região de Joinville - UNIVILLE, \\ Joinville, SC, Brasil 89219-710 \\ ${ }^{2}$ Programa de Pós-Graduação em Ciência e Engenharia de Materiais - PGMAT), \\ Laboratório de Materiais Vitrocerâmicos - VITROCER, Universidade Federal de Santa Catarina - UFSC, \\ Florianópolis, SC, Brasil 88040-900 \\ *fraupp.pereira@gmail.com
}

\begin{abstract}
Resumo
O desenvolvimento de atividades de valorização de resíduos sólidos pode viabilizar a produção de produtos industriais com elevado potencial de reaproveitamento como fonte alternativa mineral. Atualmente, os resíduos sólidos industriais tornaram-se uma importante linha de pesquisa na área de Engenharia. Apesar disso, poucos estudos têm sido realizados com interação efetiva de parâmetros ambientais, econômicos e sociais que demonstram aplicações destas matérias-primas pela indústria de transformação. Neste trabalho são apresentados e discutidos os resultados de investigação relacionados à valorização de um resíduo sólido industrial (casca cerâmica) gerado no processo de fundição de precisão por cera perdida. Este material apresenta características muito interessantes e adequadas à produção de filtros cerâmicos refratários. Desta forma, cerâmicos porosos foram obtidos através do processo da réplica poliméricae o seu desempenho testado industrialmente na filtragem de ferro fundido no estado líquido (1,76 $\mathrm{kg} / \mathrm{s}$ ). Os componentes metálicos produzidos apresentaram excelente acabamento superficial.

Palavras-chave: valorização de resíduos, casca cerâmica, filtros cerâmicos refratários.
\end{abstract}

\begin{abstract}
The activities development of valorization of solid waste can facilitate the production of industrial products with high reuse potential as mineral alternative source. Currently, the industrial solid wastes have become an important line of research in Engineering. Nevertheless, few studies have been conducted with effective interaction of environmental, economic and social parameters that demonstrate applications of these raw materials in the manufacturing industry.In this paper are presented and discussed the research results related to the valorization of a solid industrial waste (ceramic shell) generated in the investment casting by lostwax process. This material has very interesting features and suitable for the production of refractory ceramic filters. Thus, porous ceramics were obtained by the method of the replica polymeric and their performance tested in industrial filtering of cast iron liquid $(1.76 \mathrm{~kg} / \mathrm{s})$. The produced metals components showed excellent surface finish.

Keywords: valorization of waste, ceramic shell, refractory ceramic filters.
\end{abstract}

\section{INTRODUÇÃO}

Espumas cerâmicas ou cerâmicas porosas celulares podem ser produzidas pelo processo da réplica polimérica [1], também chamado de processo de replicação [2]. Este processo consiste na impregnação de uma esponja polimérica com uma suspensão cerâmica, seguido por um tratamento térmico que elimina o corpo orgânico (esponja) e sinteriza o esqueleto cerâmico na forma de uma réplica do corpo polimérico impregnado. Cerâmicas porosas celulares estão comercialmente disponíveis para uma ampla gama de aplicações tecnológicas incluindo filtros, membranas, substratos catalíticos, isolamento térmico, meios de queimador de gás, materiais refratários e painéis estruturais leves [3-6]. Nestes casos, cerâmicas porosas são utilizadas devido às suas propriedades funcionais, como o baixo coeficiente de expansão térmica e condutividade térmica, bem como elevada permeabilidade e inércia química [6]. Considerando-se as particularidades específicas de cada aplicação, cerâmicas porosas são consolidadas através da utilização de diversos tipos de materiais, incluindo cordierita, mulita, carbeto de silício, alumina, zircônia parcialmente estabilizada, materiais de base de argila, alguns sistemas compósitos, bem como resíduos sólidos [7-14].

A valorização de resíduos para a produção de novos materiais tem atraído grande interesse da sociedade e dos 
cientistas em particular [15], uma vez que representam forte potencialidade e viabilidade de aplicação como interessantes fontes alternativas de matérias-primas. Porém, estes materiais ainda são frequentemente negligenciados por simples ineficiência de gestão tecnológica produtiva e ambiental, do fato de apresentarem similariedades em composição às matérias-primas habitualmente utilizadas em diversos setores da indústria de transformação. Ressaltase que para além da compatibilidade de componentes, os resíduos podem muitas vezes trazer benefícios ao processo de fabricação de novos produtos, como economia energética inserida em operações anteriores no processo gerador deste material/subproduto industrial. Muitos destes resíduos apresentam grande potencial de valorização e merecem estudos de investigação e de sistematização experimental $[14,16]$.

O subproduto gerado a partir do processo de fundição de cera perdida utilizado na produção de peças metálicas inserese perfeitamente neste contexto, sendo caracterizado por um nobre material refratário composto mineralogicamente de mulita $\left(3 \mathrm{Al}_{2} \mathrm{O}_{3} \cdot 2 \mathrm{SiO}_{2}\right)$ e silicato de zircônio $\left(\mathrm{ZrSiO}_{4}\right)$. Assim, a função deste material para atuação em processos térmicos de alta temperatura foi investigado com base no sistema ternário $\mathrm{ZrO}_{2}-\mathrm{SiO}_{2}-\mathrm{Al}_{2} \mathrm{O}_{3}(\mathrm{ZSA})$. Em termos de composição química, o óxido dominante é a alumina $\left(\mathrm{Al}_{2} \mathrm{O}_{3}\right)$, seguido por sílica $\left(\mathrm{SiO}_{2}\right)$, óxido de zircónio $\left(\mathrm{ZrO}_{2}\right)$ e finalmente, por óxidos de ferro e minoritários (alcalinos). Embora que em processamentos normais defabrição, o equilíbrio termodinâmico geralmente não seja atingido, o diagrama de fase do sistema ASZ pode ser utilizado para prever tendências de reações e adequação de composições, assim como, para a definição de parâmetros de processamento. Entre as aplicações que podem ser viabilizadas para o desenvolvimento de produtos a partir de resíduos, considerase, em especial, a fabricação de filtros para metais fundidos [17].

Filtros para aplicação em altas temperaturas devem ter elevada permeabilidade e resistência mecânica, bem como tamanho de poros e interconectividade adequados ao tipo de metal a ser filtrado [18]. São produtos que possuem a função de reter quaisquer inclusões de escória e/ou areia, e também o controle do fluxo de metal líquido para dentro da cavidade do molde [19]. Neste estudo, desenvolveram-se filtros cerâmicos refratários produzidos pelo reaproveitamento mineral de um resíduo industrial (casca cerâmica) em laboratório e que posteriormente foi testado em escala industrial.

\section{MATERIAIS E MÉTODOS}

Com base em estudos metodológicos para a qualificação de resíduos sólidos como materiais alternativos de Engenharia [20], estabeleceu-se primeiramente um plano de amostragem para o resíduo em estudo (NBR 10007/04) de forma a garantir a representatividade necessária para 0 desenvolvimento da proposta de valorização. A classificação de periculosidade da casca cerâmicaseguiu a metodologia especificada na NBR 10004/04 (Classificação de Resíduos Sólidos). O resíduo cerâmico, tal como gerado (após processo de fundição - quebra da casca) foi caracterizado com um tamanho médio de $5 \mathrm{~cm}$, cor acinzentada e baixíssimo teor de umidade $(<0,6 \%)$. Este material foi triturado num almofariz de aço (redução $<1 \mathrm{~cm}$ ) e, em seguida, submetido à moagem a úmido num moinho e bolas de alumina durante 90 min. A distribuição média do tamanho das partículas do pó resultante foi determinada utilizando um analisador de tamanho de partícula de dispersão a laser (Master Sizer 2000, Malvern). A caracterização química foi realizada por espectroscopia de fluorescência de raios X (FRX, Philips PW 2400) e a investigação das fases cristalinas dos resíduos e das amostras sinterizadas um difratômetro de raios X com radiação Cuk $\alpha$ (Philips X'Pert). Com base no diagrama ternário do sistema $\mathrm{ZrO}_{2}-\mathrm{SiO}_{2}-\mathrm{Al}_{2} \mathrm{O}_{3}(\mathrm{ZSA})$, foi formulada uma composição combinando-se o resíduo casca cerâmica (87,5\%) e caulim (12,5\%) em massa de sólidos secos, denominada de composição FC (Filtro Cerâmico). A Fig. 1 mostra de forma representativa a localização da composição FC, do resíduo cerâmico e do caulim no sistema ternário ZSA.

Com a composição $\mathrm{FC}$, foram preparadas suspensões cerâmicas com $80 \%$ de sólidos, $20 \%$ de água e $1,5 \%$ de dispersante (Darvan 821A, RT Vanderbilt Co.) calculado com base na massa de sólidos secos. O caulim foi adicionado à composição como agente suspensor (tratamento reológico) e também mineralizador (tratamento térmico). A produção dos filtros de cerâmica foi realizada imergindo esponjas de poliuretano (INOAC Corporation - Japão) com dimensões de $50 \mathrm{~mm} \times 50 \mathrm{~mm} \times 20 \mathrm{~mm}$ e densidade nominal de poro de 10 ppi (poros por polegada) em suspenões cerâmicas adequadas a este processo. Estas esponjas foram utilizadas

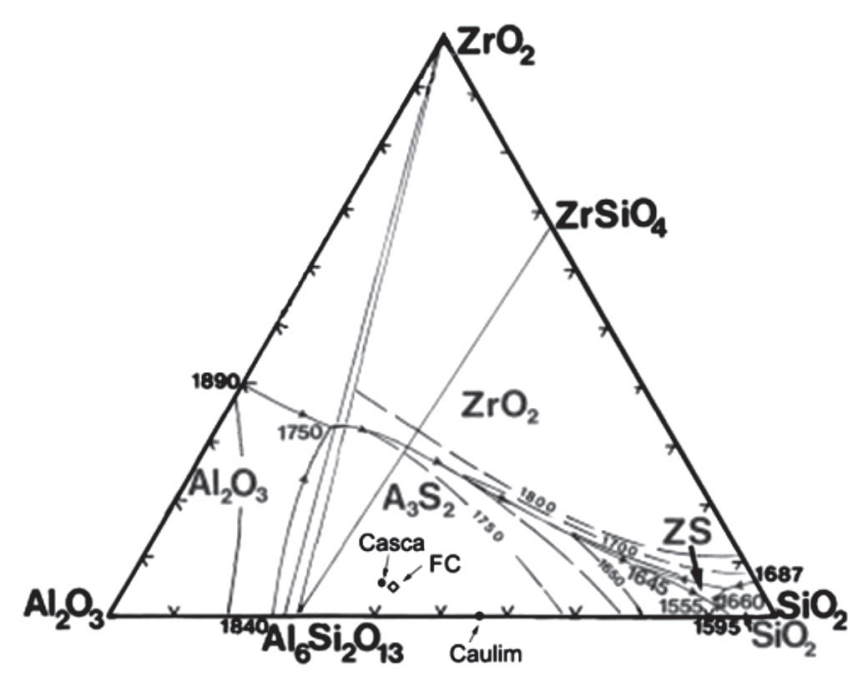

Figura 1: Localização da composição formulada (Filtro Cerâmico FC), do resíduo (casca cerâmica) e do Caulim no diagrama de fases do sistema ternário $\mathrm{ZrO}_{2}-\mathrm{SiO}_{2}-\mathrm{Al}_{2} \mathrm{O}_{3}(\mathrm{ZSA})$.

[Figure 1: Location of the formulated composition (Ceramic Filter - FC) of the waste (ceramic shell) and kaolin in the phase diagram of the ternary system $\mathrm{ZrO}_{2}-\mathrm{SiO}_{2}-\mathrm{Al}_{2} \mathrm{O}_{3}(\mathrm{ZSA})$.] 
Tabela I - Composição química (FRX) das matérias-primas utilizadas. PF: Perda ao Fogo. [Table I - Chemical composition (XRF) of the raw materials used. PF: Loss Fire.]

\begin{tabular}{cccccccccc}
\hline \multirow{2}{*}{ Matérias-primas } & \multicolumn{8}{c}{ Composição química (\%) } \\
\cline { 2 - 10 } & $\mathrm{Al}_{2} \mathrm{O}_{3}$ & $\mathrm{SiO}_{2}$ & $\mathrm{ZrO}_{2}$ & $\mathrm{TiO}_{2}$ & $\mathrm{Fe}_{2} \mathrm{O}_{3}$ & $\mathrm{~K}_{2} \mathrm{O}$ & $\mathrm{Na}_{2} \mathrm{O}$ & Outros & $\mathrm{PF}$ \\
\hline Casca cerâmica & 53,3 & 36,2 & 5,4 & 1,4 & 1,7 & 0,9 & 0,4 & $<0,7$ & - \\
Caulim & 39,9 & 50,1 & - & - & 0,5 & 0,9 & - & $<0,3$ & 8,3 \\
\hline
\end{tabular}

como réplica e imersas três vezes com intervalos de 10 min. Posteriormente, as amostras de esponja impregnadas foram secas à temperatura ambiente $\left(25^{\circ} \mathrm{C}\right)$ durante $24 \mathrm{~h}$. O estudo reológico detalhado pode ser observado em trabalho anterior [15].

A fim de determinar o comportamento térmico da composição FC, secou-se a suspensão preparada e o pó resultante desta operação foi compactado uniaxialmente a $100 \mathrm{MPa}$ em cilíndros de $10 \mathrm{~mm}$ de diâmetro e $6 \mathrm{~mm}$ de espessura. Os corpos obtidos foram secos e sinterizados em um forno elétrico (Jung CPM 45) com taxa de aquecimento de $10{ }^{\circ} \mathrm{C} / \mathrm{min}$ numa faixa de temperatura entre 1500 e 1650 ${ }^{\circ} \mathrm{C}$ com tempo de patamar na zona de máxima temperatura de $2 \mathrm{~h}$. Os parâmetros de tratamento térmico (temperatura e tempo) foram estabelecidos como condição para a obtenção de materiais densos. Esta condição também foi apoiada por meiodo caráter refratário revelado pelas análises das matériasprimas constituintes (Tabela I, FRX) e pelo consequente posicionamento no triângulo de compatibilidade $\mathrm{A}_{3} \mathrm{~S}_{2}-\mathrm{ZS}-\mathrm{S}$. A posição da composição $\mathrm{FC}$ neste sistema indica o início do amolecimento no campo preliminar da fase mulita, $\mathrm{Al}_{6} \mathrm{Si}_{2} \mathrm{O}_{13}\left(\mathrm{~A}_{3} \mathrm{~S}_{2}\right.$, na forma simplificada) por volta de $1550{ }^{\circ} \mathrm{C}$.

$\mathrm{O}$ tratamento térmico das esponjas impregnadas e secas deu-se a uma taxa de aquecimento de $0,5{ }^{\circ} \mathrm{C} / \mathrm{min}$ até $500{ }^{\circ} \mathrm{C}$ (patamar de $1 \mathrm{~h}$ - degradação térmica da esponja polimérica), seguido pela pré-sinterização a uma taxa de aquecimento de $0,5^{\circ} \mathrm{C} / \mathrm{min}$ até $1200^{\circ} \mathrm{C}$ (patamar de $1 \mathrm{~h}$ ) e, finalmente, a sinterização $\left(1500-1650^{\circ} \mathrm{C}\right.$ durante $\left.2 \mathrm{~h}\right)$ a uma taxa de aquecimento de $5^{\circ} \mathrm{C} / \mathrm{min}$ para a consolidação das espumas cerâmicas (filtros). As taxas de resfriamento foram estabelecidas pela perda natural de calor do forno.

A retração da composição $\mathrm{FC}$ foi avaliada com medições dos diâmetros dos cilindros sinterizados (precisão $\pm 0,01$ $\mathrm{mm}$ ). As medidas de densidade aparente dos compactos sinterizados da composição FC foram obtidas pela aplicação do princípio de Arquimedes em água destilada a $25^{\circ} \mathrm{C}$ em uma balança analítica (Shimadzu AX200, precisão $\pm 0,001$ g). As demais massas volúmicas foram obtidas através da determinação da densidade real do pó por picnometria a hélio (Quantachrome Ultrapycnometer 1000) e a densidade relativa pela razão entre a densidade aparente (compactos sinterizados) ou geométrica (espuma cerâmica - filtro) e a densidade real do pó (composição FC). A determinação da porosidade deu-se pela diferença desta com base relativa à densidade realdo sólido da composição FC $\left(1-\rho_{\text {rel }}\right)$.

A estrutura celular dos filtros cerâmicos (espumas), assim como as próprias esponjas PU (réplicas) foram fotografadas e as imagens digitalizadas eanalisadas (software, J Imagem).
A densidade de poros em linhas de teste $(25,4 \mathrm{~mm}$ de comprimento) e o número de poros por unidade de área foram observados.Os fragmentos dos filtros produzidos também foram analisados num microscópio eletrônico de varredura (JSM-6390LV) de tal modo que, a partir das micrografias obtidas, foi possível observar o aspecto da microestrutura resultante.

A resistência à compressão foi determinada de acordo com ASTM C133 pela média de cinco corpos de prova (20 $\mathrm{mm} \times 20 \mathrm{~mm} \times 20 \mathrm{~mm}$ ), utilizando uma máquina universal de ensaios mecânicos (DL EMIC 2000), com velocidade de carregamento de $1,3 \mathrm{~mm} / \mathrm{min}$ e com auxílio de borracha semirrígida (posicionada sobre as superfícies das amostras) para eliminar os efeitos da carga localizada.

Os filtros produzidos em laboratório foram posteriormente testados numa planta industrial para a fabricação de peças de ferro fundido cinzento (fundição em moldes de areia), que normalmente utiliza filtros refratários de carboneto de silício de 7 ppi. Neste teste a capacidade de filtração foi determinada tomando o peso conjunto (ferro fundido cinzento) após a solidificação do metal e o tempo (usando um cronómetro) para encher o molde com metal fundido.

\section{RESULTADOS E DISCUSSÃO}

A casca cerâmica estudada apresenta tamanho médio de $5 \mathrm{~cm}$ (Fig. 2a, molde cerâmico quebrado) e baixíssimo percentual de umidade $(0,1 \%)$. O resíduo sólido industrialfoicominuido em almofariz (Fig. 2b), processado em moinho (Fig. 2c) e, posteriormente, caracterizado quimicamente e classificado como não perigoso (classe II). Ensaios de lixiviação realizados de acordo com a NBR 10005/04 revelam ausência de agentes corrosivos, reativos e tóxicos. Estes resultados foram determinantes para a utilização deste resíduo como fonte alternativa mineral neste trabalho. $\mathrm{O}$ caráter de corrosividade dentro dos limites estabelecidos pela norma ABNT NBR 10004:2004 foi caracterizado pela determinação do $\mathrm{pH}$ em 7,47 (na proporção de 1:1 em massade água e sólido). Já a não reatividade, foi verificada pela ausência de íons cianeto e sulfeto acima do estabelecido. Por fim, os parâmetros analisados no extrato lixiviado obtido não ultrapassaram os limites máximos permitidos para toxicidade. Portanto, este resíduo é classificado como não perigoso (Classe II) para todos os ensaios citados, sendo também inerte (Classe II-b) já que não sofre qualquer tipo de alteração em sua composição com o passar do tempo. A composição química do resíduo e do caulim é apresentada na Tabela I. A partir da FRX é possível observar que a cas- 

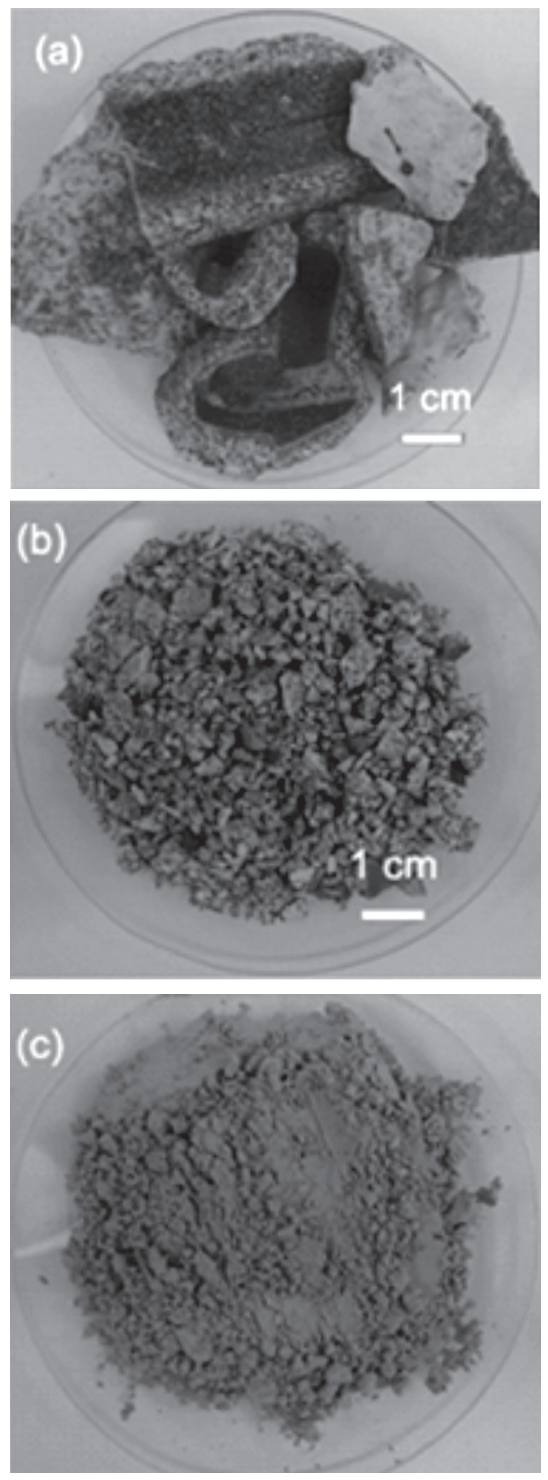

(d)

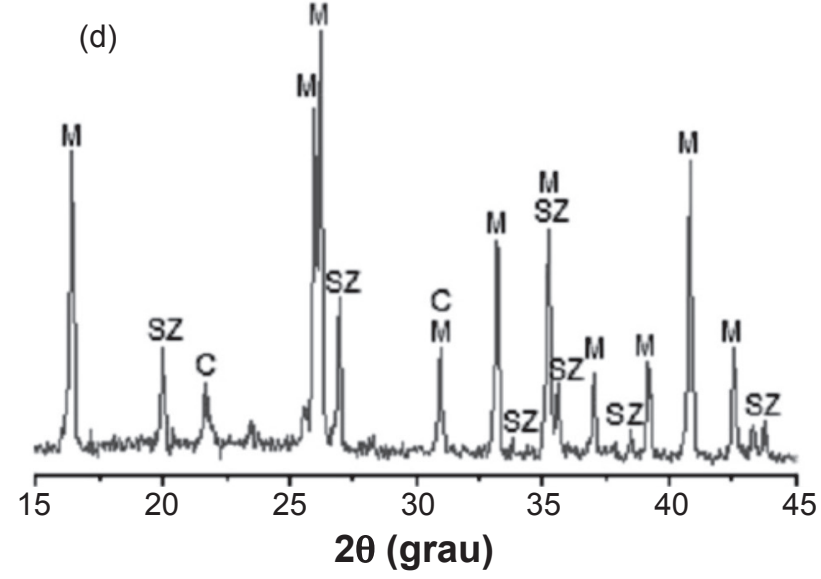

Figura 2: Fotografias da casca cerâmica (a) no estado como gerada; (b) triturada em um almofariz (c); processada em moinho. Difratograma de raios X (d), sendo M - Mulita, SZ - Silicato de Zircônio e C - Cristobalita.

[Figure 2: Photographs of the ceramic shell (a) in the generated state; (b) grinded in pestle (c); processed in mill. X-ray diffraction (d), where $M$ - Mullite, SZ - zirconium silicate, C - Cristobalite.]
Tabela II - Diâmetros característicos d10, d50 e d90 de partículas para a casca cerâmica (resíduo) e o caulim.

[Table II - Characteristic diameters d10, d50 and d90 of particles for ceramic shell (waste) and kaolin.]

\begin{tabular}{cccc}
\hline \multirow{2}{*}{ Matérias-primas } & \multicolumn{3}{c}{ Tamanho de partícula $(\mu \mathrm{m})$} \\
\cline { 2 - 4 } & $\mathrm{d}_{10}$ & $\mathrm{~d}_{50}$ & $\mathrm{~d}_{90}$ \\
\hline Casca cerâmica & 1,09 & 5,23 & 19,45 \\
Caulim & 1,29 & 6,44 & 31,65 \\
\hline
\end{tabular}

ca cerâmica é constituída principalmente de $\mathrm{Al}_{2} \mathrm{O}_{3}, \mathrm{SiO}_{2} \mathrm{e}$ $\mathrm{ZrO}_{2}$ e de outros óxidos em pequenas quantidades presentes nas matérias-primas usadas no processo de fundição.

O diâmetro de partículas da casca cerâmica após processo de moagem e do caulim comercial são dados na Tabela II. O tamanho médio de partícula (d50) para ambos os pós está dentro do intervalo de 5 a $7 \mu \mathrm{m}$, sendo inferiores a $50 \mu \mathrm{m}$. Esta estreita distribuição de tamanhos de partícula pode ser um aspecto facilitador do sistema reológico e do processo deimpregnação de esponjas poliméricas, bem como para um melhor controle do processamento térmico das espumas cerâmicas.

A Fig. $2 d$ revela a constituição mineralógica, difratograma de raios $\mathrm{X}$ da casca cerâmica, com a presença de mulita (JCPDS 01-079-1454), de silicato de zircônio (JCPDS 01084-1560) e de cristobalita (JCPDS 01-082-0512). A mulita $\left(3 \mathrm{Al}_{2} \mathrm{O}_{3} .2 \mathrm{SiO}_{2}\right)$ constitui a principal fase deste resíduo com características de alto ponto de fusão, resistência a altas temperaturas e boa estabilidade térmica e química. Além disso, destaca-se a potencialidade refratária deste material pela elevada dificuldade de síntese desta fase.

A Fig. 3 mostra curvas de tensão de cisalhamento $\tau$ em função do gradiente de velocidade $\gamma$ no estudo reológico realizado com suspensões preparadas com $80 \%$ de sólidos ediferentes quantidades de dispersante. Observa-se um comportamento reológico dilatante, o qual exibe um aumento da viscosidade com a elevação da velocidade de cisalhamento, típico de suspensões cerâmicas altamente concentradas. A presença do caulim nas suspensões estudadas acaba por proporcionar o aumento da viscosidade devido a maior interação entre as partículas e a consequente elevação da desordem do sistema reológico.

A partir da análise das curvas de fluxo pode-se observar que a menor viscosidade da suspensão $\tau / \gamma$ é obtida com a adição de $0,5 \%$ de dispersante e que para as outras adições de dispersante a viscosidade torna-se maior. Durante a impregnação das esponjas, a suspensão preparada com 1,5\% de dispersante (Darvan) apresenta a melhor capacidade de revestimento e fixação da espuma polimérica. Da mesma forma, quando a viscosidade da suspensão é muito baixa (suspensões com $0,5 \%$ de dispersante), o recobrimento dos filamentos poliméricos não se deu de maneira homogênea, resultando em materiais frágeis e também no colapso destes durante a sinterização.

Observou-se durante a preparação das suspensões que aquelas com $1,5 \%$ de dispersante permaneceram estáveis por períodos mais longos, sem a ocorrência visível de sedimen- 

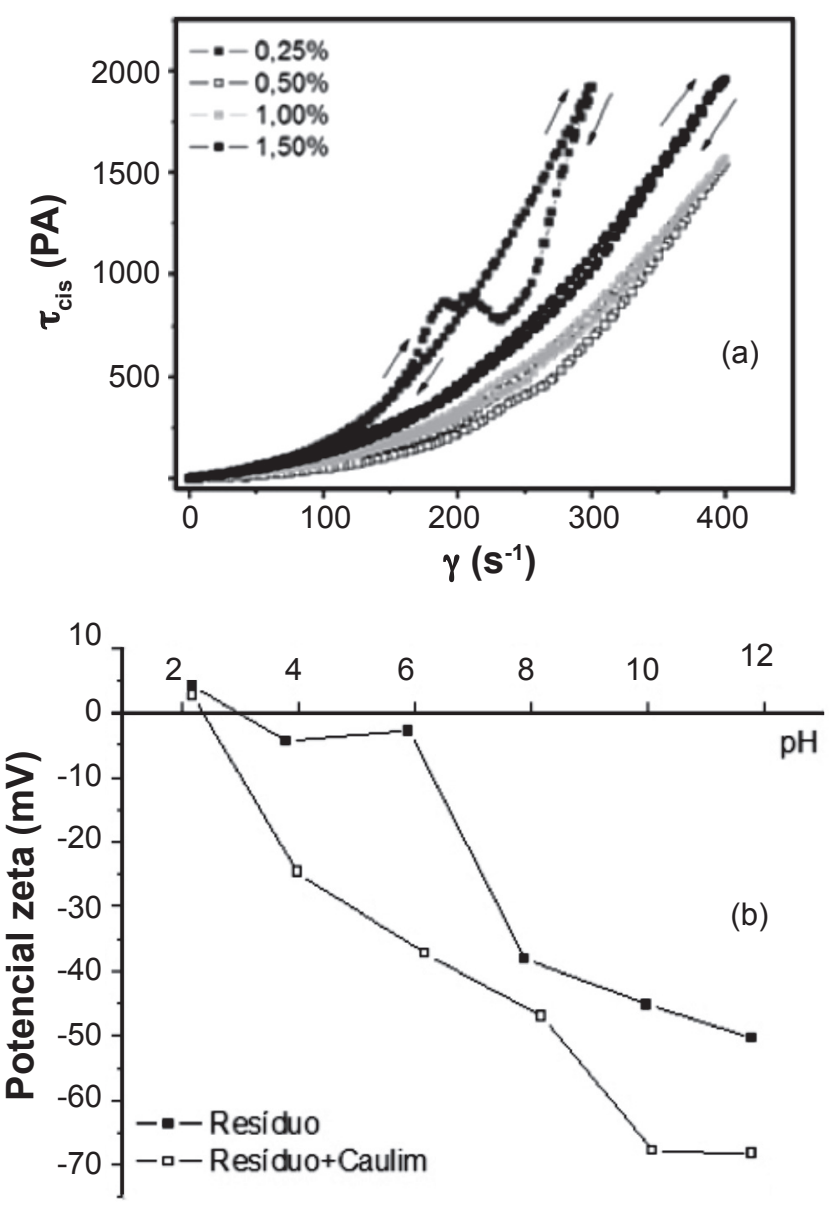

Figura 3: (a) Curvas de fluxo: tensão de cisalhamento $\tau$ versus gradiente de velocidade $\gamma$ para suspensões com $80 \%$ de sólidose diferentes porcentuais de dispersante. (b) Valores do potencial zeta em função do $\mathrm{pH}$ para o resíduo e para o resíduo e caulim.

[Figure 3: (a) Flow curves: shear stress $\tau$ versus speed gradient $\gamma$ for suspension with $80 \mathrm{wt}$.\% solids and different percentages of dispersant. (b) Zeta potential as a function of the $\mathrm{pH}$ to the residue and the residue and kaolin.]

tação de partículas. Esta observação pode ser resultado do uso de dispersantes em excesso formando aglomerados e aumento da viscosidade quando o comprimento da molécula do aditivo torna-se maior ou similar à distância entre as partículas. Considerando-se que as suspensões apresentaram valores de $\mathrm{pH}$ de 8,0 para aquelas preparadas com o resíduo e de 7,5 para aquelas com resíduo e caulim, medidas de potencial zeta (Fig. $3 b)$ foram realizadas em função do $\mathrm{pH}$. Estes resultados evidenciam que a adição de caulim aumenta a estabilidade das suspensões e comprovam a sua forte contribuiçãoao sistema reológico constituído.

As condições de tratamento térmico da composição FC foram obtidas pela análise da densidade relativa e retração diâmetral apresentada em função da temperatura de sinterização (Fig. 4a). Dentro da faixa de 1500 a $1650{ }^{\circ} \mathrm{C}$ a composição atingiu valores de máximos de densificação a $1550{ }^{\circ} \mathrm{C}$. Pode-se observar o aumento da porosidade (ilustrada por pequenas janelas de imagens de microscopia eletrônica de varredura) dos compactos de pó da composição $\mathrm{FC}$ com
- $\Theta$-Retração diâmetral —Densidade Relativa (Porosidade)
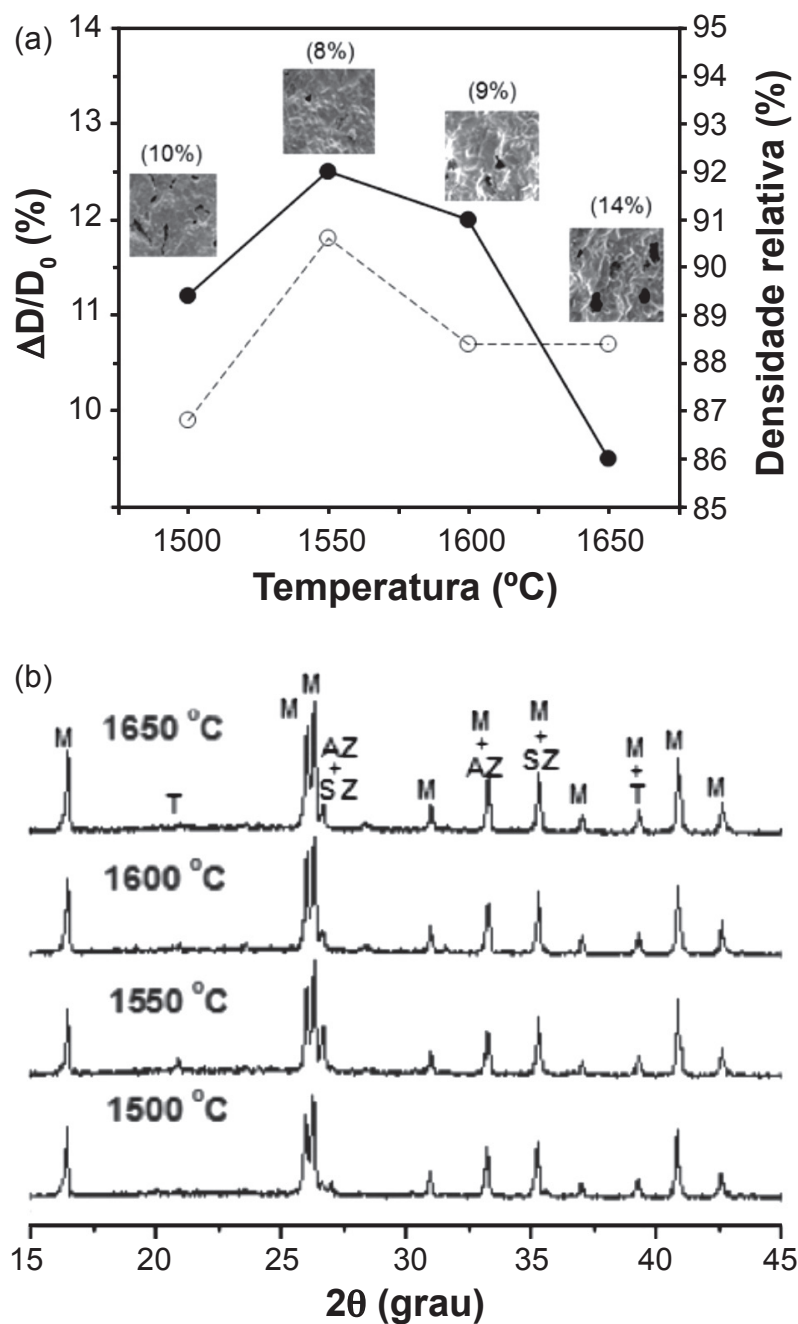

Figura 4: (a) Densidade relativa $\rho_{\text {rel }}$ e retração diametral $\Delta \mathrm{D} / \mathrm{D}_{0}$ de compactos de pós da composição $\mathrm{FC}$ em função da variação da temperatura durante $2 \mathrm{~h}$. Em detalhe são apresentadas micrografias (MEV) com valores de porosidade associados aos pontos experimentais. (b) Difratogramas de raios $\mathrm{X}$ de amostras equivalentes: M - Mulita, AZ - Aluminato de Zircônio, SZ - Silicato de zircônio, T - Tridimita.

[Figure 4: (a) Relative density $\rho_{\text {rel }}$ and diametral shrinkage $\Delta D$ / $D_{0}$ of the powder compact of FC composition as a function of the variation of temperature for $2 \mathrm{~h}$. In detail are presented micrographs (SEM) with porosity values associated with the experimental points: (b) X-ray diffraction patterns of the equivalent sample: $M$ - Mullite, AZ - Zirconium Aluminate, SZ - Zirconium Silicate, T Tridymite.]

a variação da temperatura. A menor porosidade obtida foi de $8 \%$ em $1550{ }^{\circ} \mathrm{C}$. A retração diâmetral reforça a indicação de parâmetros térmicos processuais para a mistura FC. Além disso, a presença de álcalis como $\mathrm{Na}$ e $\mathrm{K}$, assim como de ferro (Tabela I) pode contribuir para a produção de porosidade secundária a partir da expansão formação de fase vítrea a temperaturas acima de $1550{ }^{\circ} \mathrm{C}$.

O comportamento térmico da composição também foi avaliado com análises de difração de raios X (Fig. 4b), os quais revelam, além das fases cristalinas identificadas 

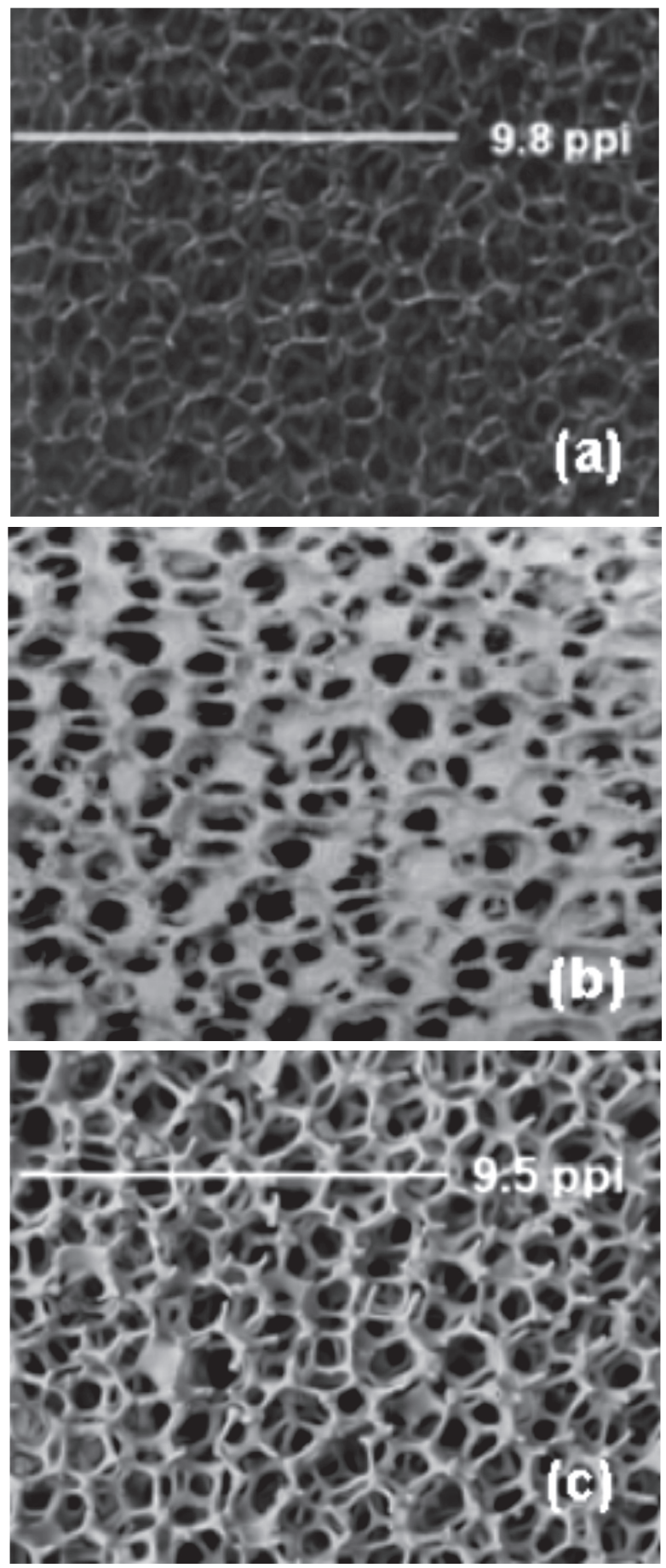

Figura 5: Fotografias digitais: (a) esponja polimérica (b) enponja impregnada com a suspensão $\mathrm{FC}$ e (c) espuma cerâmica porosa filtro FC sinterizado a $1550{ }^{\circ} \mathrm{C}$ durante $2 \mathrm{~h}$.

[Figure 5: Digital photographs (a) polymeric sponge (b) sponge impregnated with suspension $F C$ and (c) foam ceramic porous (FC filter) sintered at $1550^{\circ} \mathrm{C}$ for $\left.2 \mathrm{~h}.\right]$

nos resíduos (ver Fig. 2d), a presença do aluminato de zircónio (JCPDS 01-084-1560) e de pequenas quantidades de tridimita (JCPDS 00-003-0227). A presença de tridimita pode ser associada à transição de fases a partir da cristobalita já presente no resíduoem temperaturas mais baixas. Observou-se também o aumento na intensidade dos picos de
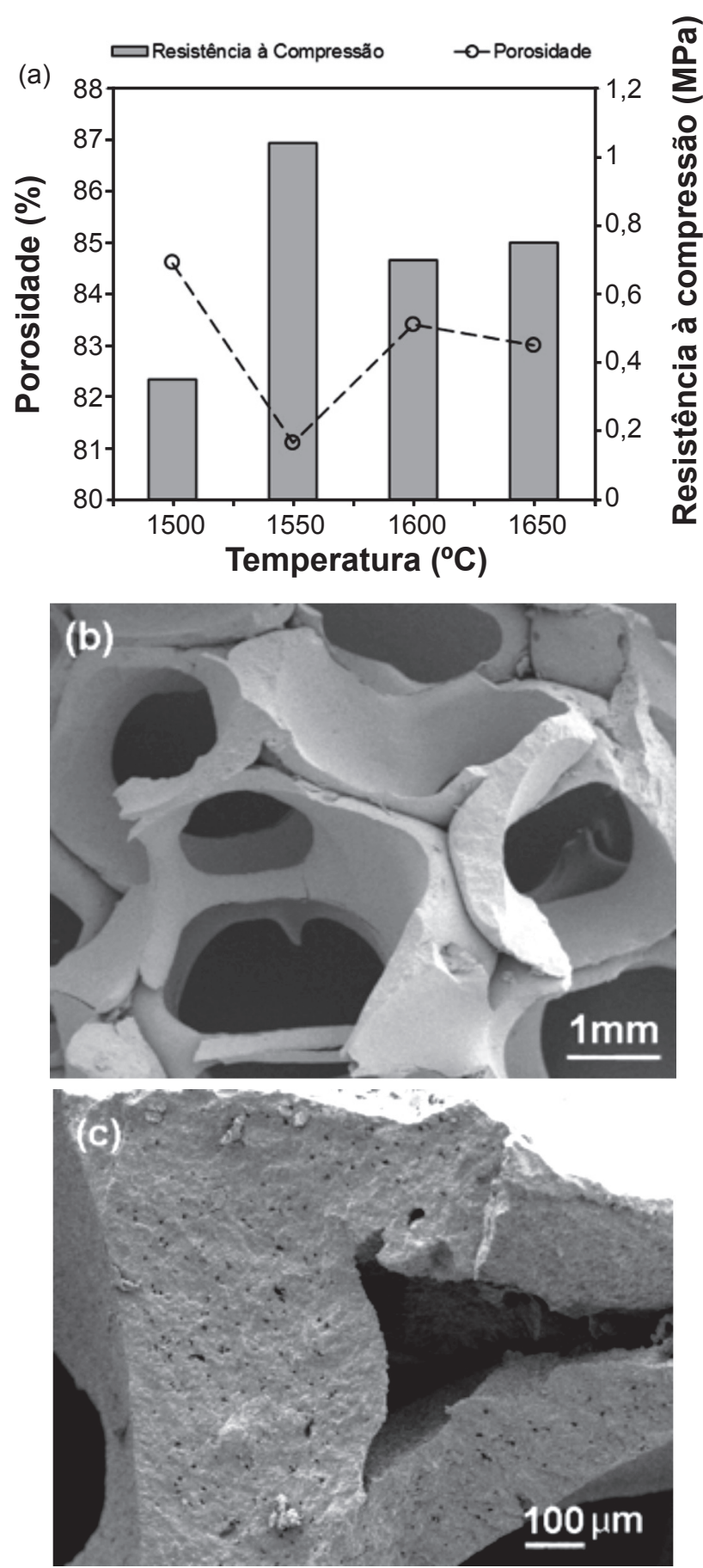

Figura 6: (a) Resistência mecânica à compressão $\left(\sigma_{c}\right)$ e porosidade $\left(1-\rho_{\text {rel }}\right)$ dos filtros cerâmicos produzidos em diferentes temperaturas de sinterização durante $2 \mathrm{~h}$. Imagens de MEV de espumas processadas a $1550^{\circ} \mathrm{C} / 2 \mathrm{~h}$ (b) com ampliação de $15 \mathrm{x}$ mostrando a superfície das cavidades e (c) pormenores dos poros presentes na estrutura celular revelados com ampliação de 100x.

[Figure 6: (a) Mechanical resistance to compression $(\sigma)$ and porosity $\left(1-\rho_{\text {rel }}\right)$ of the ceramic filters at different sintering temperatures for $2 \mathrm{~h}$. SEM images of foams processed at $1550{ }^{\circ} \mathrm{C} / 2 \mathrm{~h}$ : (b) with ampliation of $15 x$ showing the surface of the cavities and (c) details of the pores present in the cell structure with 100x magnification.]

mulita $\left(3 \mathrm{Al}_{2} \mathrm{O}_{3} .2 \mathrm{SiO}_{2}\right)$, indicando a crescente formação desta fase pela fração de caulinita $\left(\mathrm{Al}_{2} \mathrm{O}_{3} \cdot 2 \mathrm{SiO}_{2}\right)$ da composição. 
Tabela III - Avaliação da capacidade de filtração de um filtro comercial $(\mathrm{SiC})$ e do filtro FCproduzido neste estudo. Tempo (s): significa o tempo para preencher o molde com metal fundido. Peso do conjunto $(\mathrm{kg})$ : significa o peso das peças e canais após a solidificação e desmoldagem.

[Table III - Evaluation of filtration capacity of a commercial filter (SiC) and FC filter produced in this study. Time (s): means the time for filling the mold with molten metal. Setweight $(\mathrm{kg})$ : mean the weight of the parts and channels after solidification and demoulding.]

\begin{tabular}{cccc}
\hline $\begin{array}{c}\text { Tipos de } \\
\text { Filtro }\end{array}$ & $\begin{array}{c}\text { tempo } \\
(\mathrm{s})\end{array}$ & $\begin{array}{c}\text { Peso do conjunto } \\
\text { (kg) ferro } \\
\text { fundido cinzento }\end{array}$ & $\begin{array}{c}\text { Capacidade de } \\
\text { filtração }(\mathrm{kg} / \mathrm{s})\end{array}$ \\
\hline Comercial & 6,1 & \multirow{2}{*}{13,05} & 2,1 \\
\cline { 1 - 2 } & & & 1,7 \\
\hline
\end{tabular}

Desta forma, foram definidas as condições de processamento térmico das esponjas poliméricas impregnadas com a suspensão preparadas com a composição $\mathrm{FC}$ em $1550{ }^{\circ} \mathrm{C}$ com patamar de $2 \mathrm{~h}$.

A Fig. 5 mostra fotos digitais relacionadas com (a) esponja polimérica, (b) esponja impregnada com a suspensão FC e (c) a espuma cerâmica sinterizada a $1550{ }^{\circ} \mathrm{C}$ durante 2 $\mathrm{h}$, evidenciando as estruturas de poros. Nas imagens também são apresentadas medidas lineares de densidade de poros que mostram a esponja polimérica comercial (Fig. 5a) com9,8 \pm 0,9 ppi e a espuma cerâmica produzida (Fig. 5 c) com $9,5 \pm 1,1$ ppi. A diferença entre a densidade linear dos poros $(-0,3 \mathrm{ppi})$ pode ser relacionada com a contração volumétrica do corpo cerâmico poroso (filtro) após o processamento térmico.

Com o objetivo de confirmar as condições de processamento térmico obtidas pela avaliação realizada por ensaios de caracterização dos compactos de pós da composição FC (Fig. 4), espumas cerâmicas também foram caracterizadas mecanicamente após a sinterização em diferentes temperaturas (Fig. 6). Observa-se que a relação entre a resistência à compressão e a porosidade de espumas cerâmicas em diferentes temperaturas durante $2 \mathrm{~h}$ revela que amáxima resistência mecânica $(\sim 1 \mathrm{MPa})$ foi alcançada a $1550{ }^{\circ} \mathrm{C}$. Acima desta temperatura a resistência diminuiu e praticamente mantem-se constante no intervalo de $1600 \mathrm{e}$ $1650{ }^{\circ} \mathrm{C}$

A Fig. 6b revela cavidades celulares com a presença de defeitos inerentes ao processo de replicação de esponjas poliméricas. Estes defeitos podem ser originários na degradação do polímero durante o processamento térmico e/ou no processo de impregnação da esponja, uma vez que a suspensão pode formar diferentes espessuras de parede. Observa-se pela imagem da Fig. 6c que apesar da espuma sinterizada atingir um elevado grau de densificação (92\%), fica evidenciada a presença de poros nas paredes da estrutura celular produzida ( $8 \%$ de porosidade, ver Fig. 4a). No entanto, sabe-se [21] que silicato de zircônio $\left(\mathrm{ZrSiO}_{4}\right)$ funde a $1540{ }^{\circ} \mathrm{C}$, o que torna razoável a suposição de que a composição cerâmica processada a $1550{ }^{\circ} \mathrm{C}$ reage com a mulita preexistente no resíduo (casca
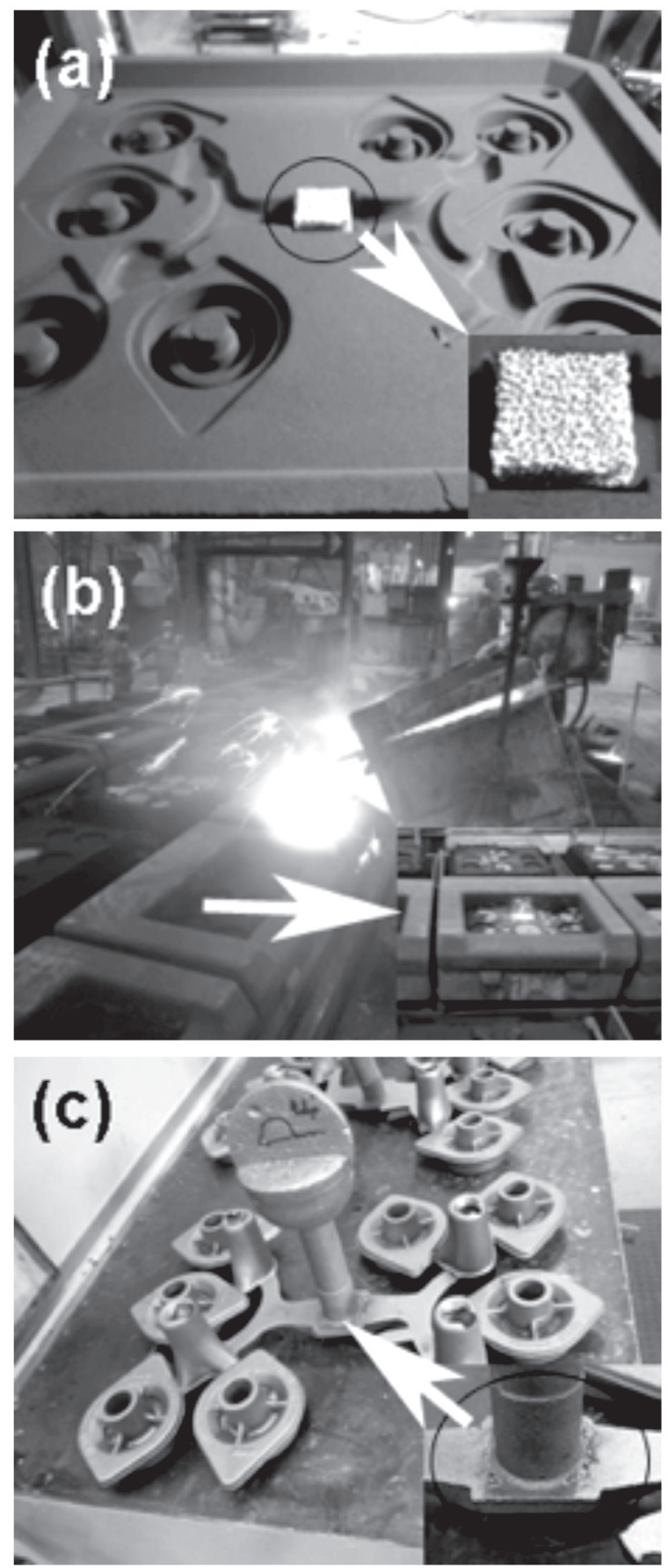

Figura 7: Fotografias mostrando as etapas de fabricação de peças de ferro fundido: (a) caixa do molde em areia usando o filtro FC produzido neste trabalho; (b) vazamento do metal líquido na cavidade do molde e (c) peças metálicas solidificadas com o detalhe do canal de alimentação domolde.

[Figure 7: Photographs showing the steps of manufacturing cast iron parts: (a) mold sand box using the FC filter produced in this work; (b) leakage the liquid metal into the mold cavity, and (c) solidifying of the metal parts with the mold feed channel in the detail.]

cerâmica) aumentando assim a densificação do filtro cerâmico FC.

A Tabela III mostra os dados industriais de desempenho do filtro cerâmico produzido no laboratório $\left(1550{ }^{\circ} \mathrm{C} / 2 \mathrm{~h}\right)$, comparativamente ao filtro comercial de carboneto de silício 
(SiC) utilizado pela empresa para a filtração de ferro fundido para a produção de peças metálicas. A capacidade de filtração determinada para o filtro FC foi um pouco menor do que aquela obtida pelo filtro comercial $(1,7 \times 2,1 \mathrm{~kg} / \mathrm{s})$. Esta diferença pode ser relacionada a densidade linear de poros do filtro comercial (7 ppi). No entanto, esta baixa capacidade de filtração pode também ser responsável pelo melhor acabamento superficial observado nas peças metálicas produzidas (Fig. 7), devido possivelmente a menor turbulência do metal líquido na cavidade do molde.

\section{CONCLUSÕES}

O resíduo (casca cerâmica) objeto da presente proposta de valorização como fonte alternativa mineral revela, através da caracterização do material, forte potencial como matéria-prima para a produção de filtros para fundição de metais, uma vez que contêm, em sua maioria, fase mineralógica como mulita e silicato de zircônio, que lhe atribuem elevada refratariedade. As suspensões cerâmicas da composição FC preparadas com $80 \%$ de sólidos, $20 \%$ de água e $1,5 \%$ de dispersante, mostraram comportamento reológico bastante adequado à impregnação de esponjas poliméricas. Estas esponjas $(9,8 \pm 0,9$ ppi) impregnados com a suspensão (composição FC) foram processadas termicamente a $1550{ }^{\circ} \mathrm{C}$ durante $2 \mathrm{~h}$, de modo que os filtros fossem bem reproduzidos dimensionalmente $(9,5 \pm 1,1$ ppi) e com propriedades mecânicas adequadas à função proposta (1,0 MPa, filtragem de metal líquido). Filtros produzidos em laboratório apresentaram excelentes resultados durante testes industriais com filtração de ferro fundido. Os cerâmicos testados mostraram uma razoável eficiência de filtragem $(1,7 \mathrm{~kg} / \mathrm{s})$ com a produção de peças metálicas livres de defeitos e excelente acabamento superficial.

\section{AGRADECIMENTOS}

Os autores agradecem a FAPESC e o CNPq (PRONEX 17431/2011-9) pelo financiamento deste trabalho.

\section{REFERÊNCIAS}

[1] J. Saggio-Woyaansky, C. E. Scottetal, "Processing of porous ceramics", Am. Ceram. Soc. Bull. 71 (1992) 1674-1682. [2] K. Schwartzwalder, A. V. Somers, "Method of making porous ceramic article", US Patent 3 (1963) 090 - 094.

[3] T. Inui, T. Otowa, "Catalytic combustion of benzene soot captured on ceramic foam matrix", Appl. Catal. 14 (1985) 8393.

[4] T. Mizrah, A. Maurer, L. Gauckler, J. P. Gabathuler, “Open pore ceramic foam as diesel particulate filter", SAE Paper 890172 (1989) SAE International, Warrendale, PA, EUA.

[5] L. Montanaro, Y.Jorand, G. Fantozzi, A. Negro, "Ceramic foams by powder processing”, J. Eur. Ceram. Soc. 18 (1998) 1339-1350.

[6] P. Colombo, E. Bernardo, "Macro- and micro-cellular porous ceramics from preceramic polymers", Comp. Sci. Technol. 63 (2003) 2353-2359.
[7] F. M. Ashby, "The mechanical properties of cellular solids", Metal. Trans. A 14 (1983) 1755-1769.

[8] R. Brezny, D. J. Green, "Mechanical behavior of cellular ceramics", in Mater. Sci. Technol. - A Comprehensive Treatment; Structure and Properties of Ceramics 11, Ed. R.W. Cahn, P. Haasen, E. J. Kramer,VCH, Weinheim, Germany (1994) 463516

[9] F. F.Lange, T. K.Miller, "Open cell low density ceramics fabricated from reticulated polymer substrates", Adv. Ceram. Mater. 2 (1987) 827-831.

[10] K. Lannguth, "Preparation of macro-porous $\mathrm{SiC}-\mathrm{Al}_{2} \mathrm{O}_{3}$ composites with polysilanes and polycarbosilanes", Ceram. Int. 21 (1995) 237-242.

[11] D. Hotza, E. Souza, C. R. Rambo, A. P. N. Oliveira, T. Fey, P. Greil, "Microstructure and properties of LZSA glass-ceramic foams", Mater. Sci. Eng. A, Structural Mater.: Properties, Microstructure and Processing 476 (2008) 89-97.

[12] M. W. Quintero Guzmán, J. A. Escobar Gutiérrez, A. V. Rey, A. F. Sarmiento Santos, C. R. Rambo, A. P. N. Oliveira, D. Hotza, "Flexible polyurethane foams as templates for cellular glass-ceramics", J. Mater. Proc. Technol. 209 (2009) 5313 5318.

[13] K. B. Mundstock, A. P. N. Oliveira, D. Hotza, S. O. Rogero, "Avaliação da biocompatibilidade de vidro e vitrocerâmica do sistema SNCP $\left(\mathrm{SiO}_{2}-\mathrm{Na}_{2} \mathrm{O}-\mathrm{CaO}-\mathrm{P}_{2} \mathrm{O}_{5}\right)$ ", Quim. Nova 35 (2012) 665-670.

[14] F.Raupp-Pereira, D. Hotza, A. M. Segadães, J. A. Labrincha, "Ceramic formulations prepared with industrial wastes and natural sub-products" Ceram. Int. 32 (2006) 173-179.

[15] A. C. Carvalho, G. G. Moraes, F. R. Cesconeto, F. Raupp-Pereira, A. P. N. Oliveira, "Development of ceramic refractory filters from alternative mineral source: rheological characterization", Mater. Sci. Forum 77 (2014) 664-669.

[16] T. Y.Yang, H. B. Ji, S. Y. Yoon, B. K. Kimb, H. C. Park, "Porous mullite composite with controlled pore structure processed using a freeze casting of TBA-based coal fly ash slurries", Resources, Conservation and Recycling 54 (2010) 816-820.

[17] W. Chi, D. Jiang, Z. Huang, S. Tan, "Sintering behavior of porous SiC ceramics", Ceram. Int. 30 (2004) 869-874.

[18] P. Colombo, E. P. Stankiewicz, "Other Developments and Special Applications", in: M. E. Scheffler, P. Colombo, Cellular Ceramics: structure, manufacturing, properties and applications, Weinheim: Wiley-VCH (2005) 596-597.

[19] A. Rudolph, I. Olson, L. C. B. Martins, "Liquid Metal Filtration”, in: M. Scheffler, P. Colombo, Cellular ceramics: structure, manufacturing, properties and applications, Weinheim: Wiley-VCH (2005) 403-404.

[20] F. Raupp-Pereira, M. J. Ribeiro, D. P. Coll, A. M. Segadães, J. A. Labrincha, "Reciclaje de residuos sólidos industriales para la producción de materiales cementíceos y refractarios", Rev. Residuos 86 (2005) 56-63.

[21] J. B. Rodrigues Neto, R. Moreno, "Effect of mechanical activation on the rheology and casting performance of kaolin/ talc/alumina suspensions for manufacturing dense cordierite bodies", Appl. Clay Sci. 38 (2008) 209-218.

(Rec. 17/12/2014, Ac. 30/04/2015) 